\title{
Methods for calculating the pressure loss of the air flow and energy consumed by the fan of the cotton harvester
}

\author{
Rafik Matchanov ${ }^{1}$, Anvar Rizaev ${ }^{2}$, A Yuldashev ${ }^{2}$, Davronbek Kuldoshev ${ }^{2 *}, M$ Mirzaeva $^{2}$ \\ ${ }^{1} \mathrm{JV}$ “Agrikhim”LLC, Tashkent, Uzbekistan \\ ${ }^{2}$ Institute of Mechanics and Seismic Stability of Structures of the Academy of Sciences of the \\ Republic of Uzbekistan, Tashkent, Uzbekistan
}

\begin{abstract}
The article presents the methods for calculating the pressure loss of the pneumatic transport system of a cotton harvester related to the power consumption of the fan drive. Mathematical models for computer calculation are presented. Numerical studies of the influence of the pipeline diameter and the air mixture composition on the power consumption of the fan drive are presented. It was determined that with an increase in the diameter of the pipeline from $0.15 \mathrm{~m}$ to $0.175 \mathrm{~m}$ and a change in the mixture concentration $\mu=0.228 \div 0.338$ for a serial receiving chamber, the fan drive power decreases from $10.3 \mathrm{~kW}$ to $5.79 \mathrm{~kW}$.
\end{abstract}

\section{Introduction}

Improving the efficiency of the pneumatic transport system of the cotton harvester while maintaining the biological qualities of cotton, with less loss of raw material and less power consumption of the fan drive, is a relevant aspect. However, no attention was paid to the development of mathematical models to effectively use the pneumatic transport system [1$15]$.

In this regard, the development of mathematical models for the pneumatic transport system of the cotton-harvesting machine is the goal of this article.

When air moves in pipelines, internal and external friction forces of its individual particles or layers arise. Air has a viscosity, which manifests itself in the form of internal friction forces induced by relative displacement of its adjacent layers. External friction forces arise from the contact of moving air particles with the pipeline walls.

When a mixture of air and raw material moves in pipes, pressure losses increase due to the need to impart acceleration to the particles of the material, to compensate for the energy consumption to lift the particles and to restore the velocity of the particles of the solid component when they interact with the wall material of the pipe and with each other.

Therefore, the pressure loss is a consequence of flow resistance (air drag).

When transporting raw cotton, there are different unfavorable areas; one of them is the pipe- bend. As noted by F.G. Zuev [16], when the flow moves along the pipe-bend, pressure losses consist of the sum of losses due to friction and air vortices at the flow turn,

* Corresponding author: don_02@mail.ru 
friction of solid particles against the material of the pipe wall, inertial forces of the flow components and velocity loss during a turn.

Aerodynamic calculations for the cotton transport in cotton harvesters XN-3,6 were given by N.A. Artykov in [17]. However, the study did not consider the different values of the pressure loss in the pipelines connected to a single fan.

Different values of pressure loss occur due to the difference in the pipeline lengths and the number of pipe-bends that connect the cotton receiving chamber to the air collector. Besides, the chamber located on the front spindle drums receives more raw cotton than the rear receiving chambers; this also affects the pressure loss. The authors of that article in the calculation method did not consider the air suction from the leaky pipeline connections.

In this regard, it is necessary to improve the calculation methods given in [18], considering the above disadvantages.

\section{Materials and Methods}

For the aerodynamic calculation of the pneumatic transport system of the cotton harvester, the following data are required: characteristics of the material, productivity, total pressure, and pipeline route (rise of height, presence of pipe-bends). It is necessary to select the concentration of the mixture, to determine the air velocity, the pressure loss in the pipeline network, and to calculate the corresponding power consumption of the fan (for 14XV.61.020.A and 14XV.61.060A harvesters).

Therefore, we determine the pressure loss in all elements of the pneumatic transport system for CHM MX-2.4, Figure 1.

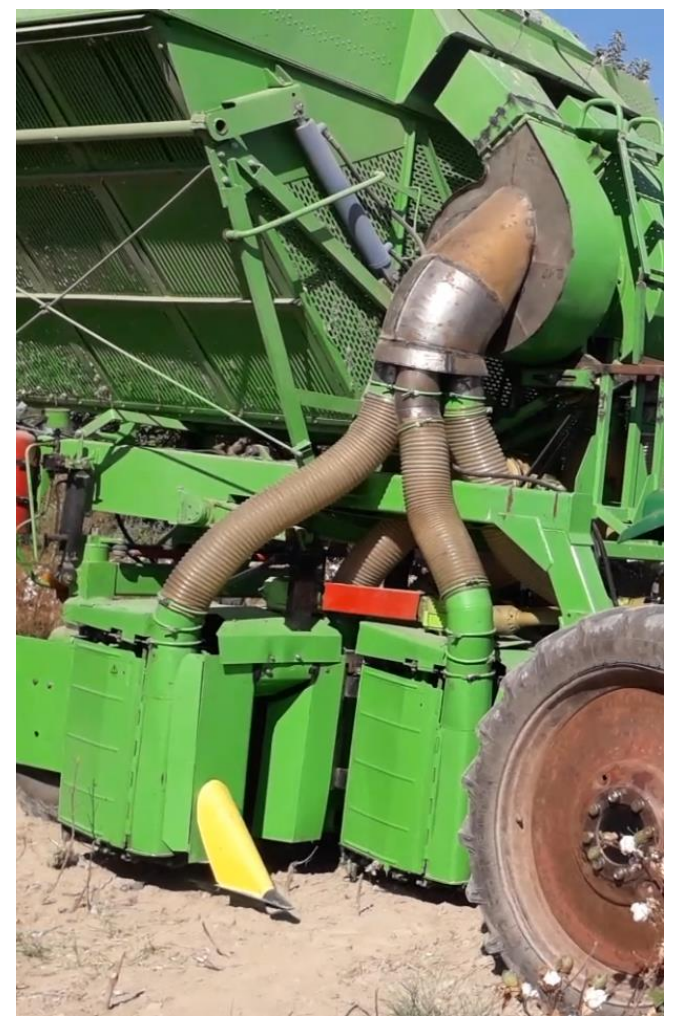

Fig. 1. Pneumatic system of the vertical-spindle cotton harvester MX-2.4 
The pressure loss at the inflow of the cotton mixture into the receiving chamber is:

$$
\Delta P_{1}=\beta \frac{\rho \vartheta^{2}}{2}
$$

where: $\vartheta-$ is the air flow velocity in the pipeline, $\mathrm{m} / \mathrm{s}$; $\rho-$ is the air density, $\left(\rho=1.29 \mathrm{~kg} / \mathrm{m}^{3}\right) ; \beta$-is the coefficient of pressure loss during acceleration of the material, $(\beta=1.8+1.5 \mathrm{~m})$ [13]

The pressure loss in the pipe-bend is determined as:

$$
\Delta P_{2}=0.5 \xi \rho \vartheta^{2}=0.5 \rho \vartheta^{2}\left(\xi_{M}+\xi_{T P}+\xi_{T}\right)
$$

where: $\xi_{M}, \xi_{T P}, \xi_{T}-$ are the resistance coefficients of the bend.

According to [17], $\xi_{M}=0.274 \xi_{T P}=0.008 \xi_{T}=\mu(0.087 \mu+0.37)$, the pressure loss in the inclined section is determined as:

$$
\Delta P_{3}=\lambda_{c m} \frac{\ell}{D} \frac{\rho \cdot \vartheta^{2}}{2}
$$

where: $\lambda_{c m}-$ is the coefficient of frictional resistance, depending on the wall roughness and the Reynolds number, and the concentration of mixture ${ }^{\mu} ; l$ and $D^{\text {are }}$ the length and diameter of the pipeline, respectively; $\rho-$ is the air density $\left(\rho=1.29 \mathrm{~kg} / \mathrm{m}^{3}\right)$; $\vartheta-$ is the velocity of conveying air, $\mathrm{m} / \mathrm{s}$ :

The resistance coefficient $\lambda_{C M}$ is determined as:

$$
\lambda_{C M}=\lambda_{T P}+\lambda_{T}^{H}
$$

According to experimental data, $\lambda_{T P}$ is determined from the number $R_{e}$ for pipeline diameters $0.15 \mathrm{~m}, 0.18 \mathrm{~m}, 0.21 \mathrm{~m}$ by the following expressions:

According to [18], $R_{e}$ is determined as:

$$
\lambda_{T P}=0.164 R_{e}^{-0,187},
$$

$$
R_{e}=\frac{\vartheta D}{v}
$$

where: $\vartheta-$ is the flow rate; $D-$ is the diameter of a circular air pipe; $v-$ is the gas kinematic viscosity coefficient;

According to [17], $\lambda_{T}^{H}$ is determined as:

$$
\lambda_{\mathrm{T}}^{\mathrm{H}}=0.025 \mu,
$$

Expression (2) has the following form

$$
\Delta P_{3}=\left(0.164 R_{e}^{-0,187}+0.025 \mu\right) \frac{\ell \cdot \rho \vartheta^{2}}{D \cdot 2}
$$

Pressurelossunder the impact at the entrance to the bend section is:

$$
\Delta P_{4}=0.5 \xi_{M} \rho \vartheta^{2}
$$


where: $\xi_{M}-$ is the coefficient of local resistance $\left(\xi_{M}=0.4\right)$.

Pressure loss at the turning section where it is connected by centrifugal fans is

$$
\Delta \mathrm{P}_{5}=0.5 \xi \rho \vartheta^{2}=0.5 \rho \vartheta^{2}\left(\xi^{1}+\xi_{\mathrm{TP}}+\xi_{\mathrm{T}}\right)
$$

where: $\xi^{1}-$ is the coefficient that takes into account the confluence of flows; according to [19], it is $\xi^{1}=0,09$.

Pressure loss in diffuser and fan outlet is:

$$
\Delta \mathrm{P}_{6}=\Delta \mathrm{P}_{\mathrm{A}}+\Delta \mathrm{P}_{\mathrm{B}}=0.5 \rho\left(\xi_{\mathrm{A}} \vartheta_{\text {inlet }}^{2}+\vartheta_{\text {outlet }}^{2}\right)
$$

where: $\xi_{д}-$ is the coefficient of resistance of the diffuser $\left(\xi_{\not}=0.008\right)[17] ; \vartheta_{\text {inlet }}^{2}-$ is the air flow velocity in the inlet section of the diffuser; $\vartheta_{\text {outlet }}^{2}-$ is the air flow velocity in the outlet section of the diffuser.

The pressure loss for lifting the material $\Delta P_{n}$ to the height $\mathrm{H}$ is determined as:

$$
\Delta P_{7}=d \rho \mu H
$$

where: $H-$ is the rise of the height of the material, $\mathrm{m} ; \Delta P_{8}-$ is the pressure loss for the suction of the pipeline system from the total volume of $k^{\prime}=1 \div 1.01$ [19].

$$
\Delta P_{8}=\left(\Delta P_{1}+\Delta P_{2}+\Delta P_{3}+\Delta P_{4}+\Delta P_{5}+\Delta P_{6}+\Delta P_{7}\right) \cdot k^{\prime} \cdot 10^{-2}
$$

Based on the above calculation method, the total pressure loss of each pipeline is determined as:

$$
\Delta P_{\text {vol } 1}=\Delta P_{1}+\Delta P_{2}+\Delta P_{3}+\Delta P_{4}+\Delta P_{5}+\Delta P_{6}+\Delta P_{7}+\Delta P_{8}
$$

We determine the average pressure loss of pipelines connected to a single fan as:

$$
\Delta P_{a v}=\frac{P_{v o l 1} \ldots \ldots P_{v o l n}}{n}, P a
$$

Determine the power consumption of the fan drive:

$$
N=\frac{\Delta P_{v o l} Q Q}{\eta \cdot 1000}=\frac{\Delta P_{v o l} \cdot Q}{0.5 \cdot 1000}, k W
$$

\subsection{Development of a mathematical model}

To develop a mathematical model for determining the power consumption of the fan drive, we assume that:

1. $Q_{1}=0.40 \div 0.55 \mathrm{~m}^{3} / \mathrm{s}$; consumed air volume of one pipeline;

2. $\mu=0 \div 0.5$; concentration ratio of the air mixture;

3. $\rho=1.29 \mathrm{~kg} / \mathrm{m}^{3}$; air density; 
4. $v=15.06 \cdot 10^{-6} \mathrm{~m}^{2} / \mathrm{s}$; coefficient of kinematic viscosity of air;

5. $D=0.15 \div 0.18 \mathrm{~m}$; diameter of a transparent flexible pipeline;

6. $\ell=1.0 \div 1.8 \mathrm{~m}$; length of the pipeline used;

7. $H=2.5 \div 3.5 \mathrm{~m}$; the rise of the height of raw cotton;

8. $\quad F_{\text {inlet }}=0.085 \mathrm{~m}^{2}$; inlet section of the delivery diffuser;

9. $F_{\text {outlet }}=0.17 \mathrm{~m}^{2}$; outlet section of the delivery diffuser;

10. $\partial=9.8 \mathrm{~m} \cdot \mathrm{s}^{-2}$; free fall acceleration;

11. $k^{\prime}=1.0 \div 1.01$; the coefficient that takes into account the suction of the pneumatic transport system;

12. $k_{1}=\frac{\Delta P_{2}}{\Delta P_{1}}=0.8 \div 1.2 ;$ the ratio of the pressure loss of the second pipeline to the first pipeline;

13. $k_{2}=\frac{\Delta P_{3}}{\Delta P_{1}}=0.8 \div 1.2 ;$ the ratio of the pressure loss of the third pipeline to the first pipeline;

14. $k_{3}=\frac{\Delta P_{4}}{\Delta P_{1}}=0.8 \div 1.2 ;$ the ratio of the pressure loss of the fourth pipeline to the first pipeline;

15. $X=1.8$; coefficient used in calculating the serial receiving chamber;

16. $X=2.65$; Coefficient used in calculating the experimental receiving chamber.

Based on the input data, we determine:

$$
\vartheta=\frac{Q_{1}}{F}=\frac{4 \cdot Q_{1}}{\pi \cdot D^{2}}=\frac{1.27 \cdot Q_{1}}{D^{2}},
$$

air flow rate in pipelines.

$Q=4 \cdot Q_{1}$; air volume in the discharge diffuser.

$\Delta P_{1}-$ is pressure loss in the receiving chambers

$$
\Delta P_{1}=(X+1.5 \mu) \frac{\rho \cdot \vartheta^{2}}{2}, \mathrm{~Pa}
$$

$\Delta P_{2}-$ is pressure loss in the bends

$$
\Delta P_{2}=0.5 \cdot \rho \cdot \vartheta^{2}[0.274+0.008+\mu(0.087 \mu+0.37)]
$$

$\Delta P_{3}-$ is pressure loss in the pipeline

$$
\Delta P_{3}=\left[0.164\left(\frac{\vartheta \cdot D}{D}\right)^{-0.187}+0.025 \mu\right] \frac{\ell \rho \vartheta^{2}}{D \cdot 2}
$$


$\Delta P_{4}-$ is pressure loss in the receiver

$$
\Delta P_{4}=0.5 \cdot 0.4 \cdot \rho \cdot \vartheta^{2}
$$

$\Delta P_{5}-$ is pressure loss in the turning section (nozzles) where it is connected to the fan

$$
\Delta P_{5}=0.5 \cdot \rho \cdot \vartheta^{2}[0.09+0.008+\mu(0.087 \mu+0.37)]
$$

$\Delta P_{6}-$ is pressure loss in the discharge diffuser

$$
\Delta P_{6}=0.5 \rho\left[0.008\left(\frac{Q}{F_{\text {inlet }}}\right)^{2}+\left(\frac{Q}{F_{\text {outlet }}}\right)^{2}\right]
$$

$\Delta P_{7}-{ }_{\text {is pressure loss when lifting material }}$

$$
\Delta P_{7}=\partial \cdot \rho \cdot \mu \cdot H
$$

$\Delta P_{8}-$ is pressure loss at the suction of the pneumatic conveying system

$$
\Delta P_{8}=\left(\Delta P_{1}+\Delta P_{2}+\Delta P_{3}+\Delta P_{4}+\Delta P_{5}+\Delta P_{6}+\Delta P_{7}\right) \cdot k^{\prime} \cdot 10^{-2}
$$

Pressure loss in four pipelines is:

$$
\begin{gathered}
\Delta P_{v o l 2}=\Delta P_{v o l 1} \cdot K_{1}, \Delta P_{v o l 3}=\Delta P_{v o l 1} \cdot K_{2}, \Delta P_{v o l 4}=\Delta P_{v o l 1} \cdot K_{3}, \\
\Delta P_{a v}=\frac{\Delta P_{v o l 1}+\Delta P_{v o l 2}+\Delta P_{v o l 3}+\Delta P_{v o l 4}}{4},
\end{gathered}
$$

$N-{ }_{\text {is consumed power of the fan drive }}$

$$
N=\frac{\Delta P_{a v} \cdot Q}{\eta \cdot 1000}=\frac{\Delta P_{a v} \cdot Q}{0.5 \cdot 1000}, K w t
$$

For the MX-1.8 cotton harvester, we determine

$$
\begin{gathered}
\Delta P_{v o l 2}=\Delta P_{v o l 1} \cdot K_{1}, \Delta P_{2 a v}=\frac{\Delta P_{v o l 1}+\Delta P_{v o l 2}}{2}, \\
Q_{2}=2 \cdot Q_{1}
\end{gathered}
$$




$$
N=\frac{\Delta P_{2 a v} \cdot Q_{2}}{\eta \cdot 1000}=\frac{\Delta P_{2 a v} \cdot Q_{2}}{0.5 \cdot 1000}
$$

\section{Results of numerical studies}

For numerical studies, we use the programs developed in [20]. To determine the effect of the concentration of the air mixture on the power consumption of the fan drive, we assume that:

Serialreceivingchamber $\left(Q_{l}=0.55 \mathrm{~m}^{3} / \mathrm{s} X=1.8, D=0.175, \mu=0 \div 0.338, H=2.5 \mathrm{~m}, l=1.0 \mathrm{~m}\right.$, $\left.k^{\prime}=1.01, k_{1}=k_{2}=k_{3}=1\right)$;resultsarepresentedinFig. 1

Experimental receiving chamber $\left(Q_{l}=0.425 \mathrm{~m}^{3} / \mathrm{s} X=2.65, D=0.175, \mu=0 \div 0.444, H=2.5\right.$ $\left.m, l=1.0 \mathrm{~m}, k^{\prime}=1.01, k_{1}=k_{2}=k_{3}=1\right)$; results are presented in Figure 2 .

To determine the influence of the pipeline diameter $(D)$ and the concentration of the air mixture $(\mu)$ on the power consumption of the drive, we assume that the initial data are:

Serial receiving chamber $\left(Q_{l}=0.55 \mathrm{~m}^{3} / \mathrm{s} X=1.8, D=0.150 \div 0.175, \mu=0 \div 0.338, H=2.5 \mathrm{~m}\right.$, $\left.l=1.0 \mathrm{~m}, k^{\prime}=1.01, k_{1}=k_{2}=k_{3}=1\right)$; results are presented in Figure 3.

Experimental receiving chamber $\left(Q_{1}=0.425 \mathrm{~m}^{3} / \mathrm{s} X=2.65, D=0.150 \div 0.175, \mu=0 \div 0.444\right.$, $\left.H=2.5 \mathrm{~m}, l=1.0 \mathrm{~m}, k^{\prime}=1.01, k_{1}=k_{2}=k_{3}=1\right)$; results are presented in Figure 4.

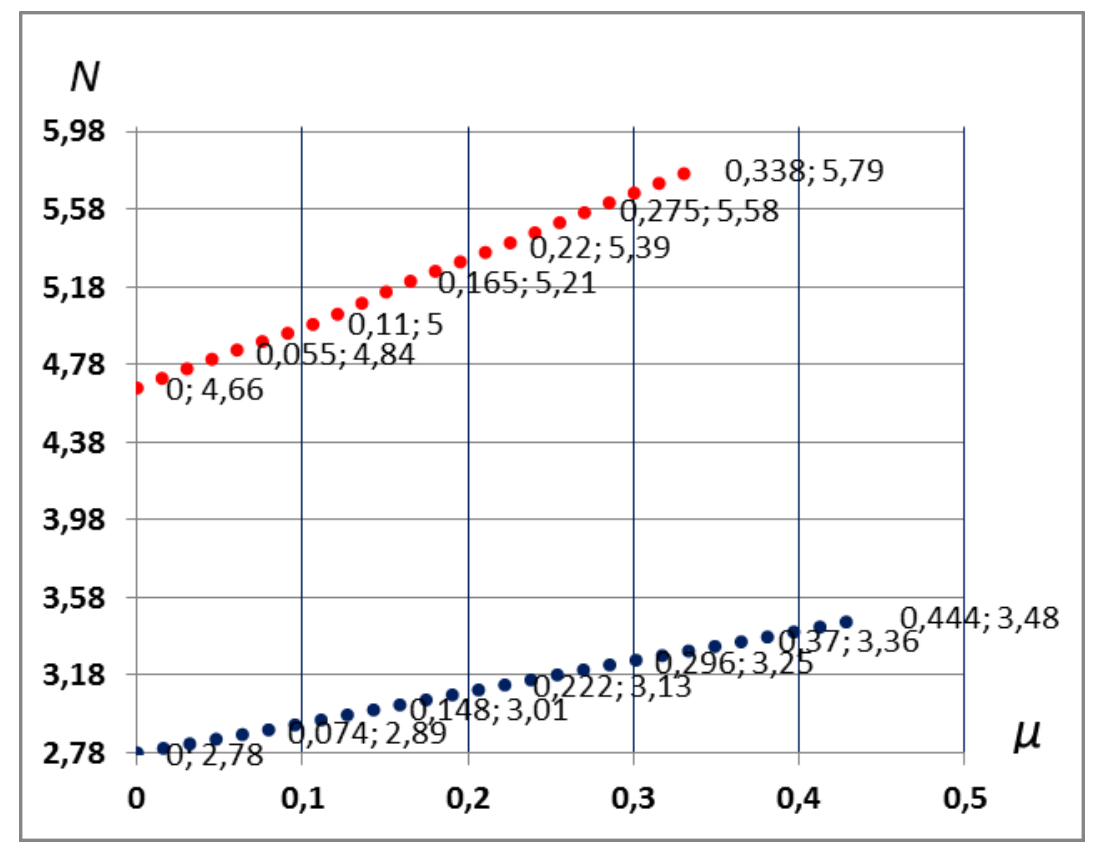

Fig. 2. Influence of the concentration of the air mixture on the power consumption of the fan drive $\mu$ $=0 \div 0.444$ of the serial and experimental receiving chambers 


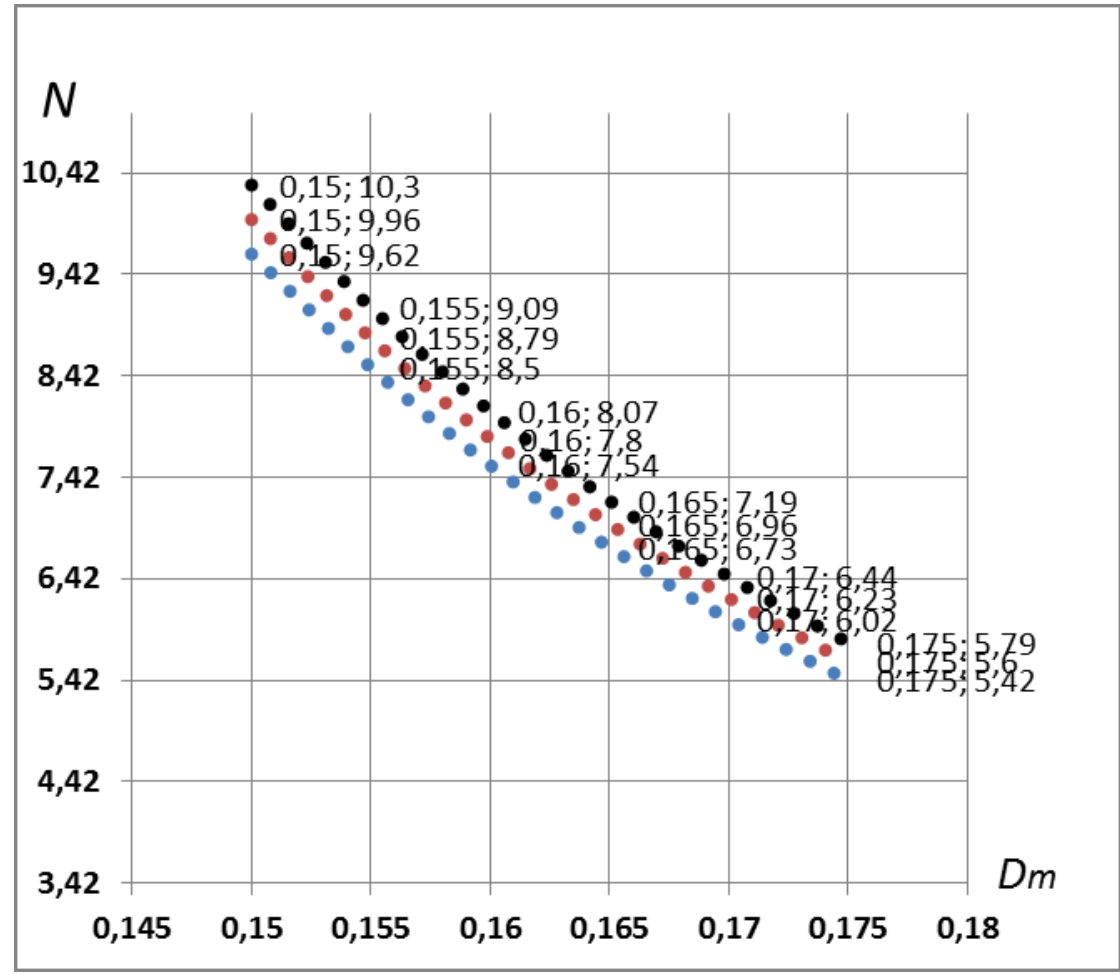

Fig. 3. Effect of change in the diameter of the pipeline and the concentration of the air mixture $(\mu)$ on the fan drive power in the serial receiving chamber

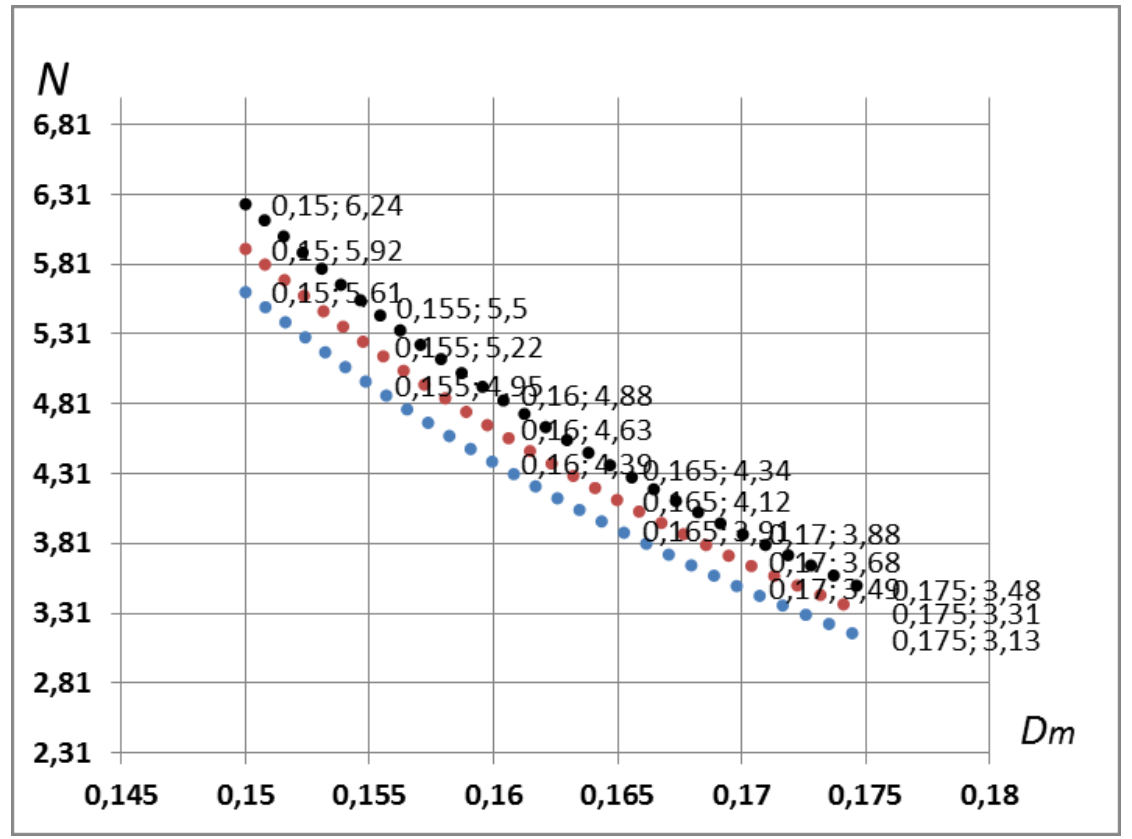

Fig. 4. Effect of change in the diameter of the pipeline and the concentration of the air mixture $(\mu)$ on the fan drive power in the experimental receiving chamber 


\section{Discussions}

From the graphs in Figure 2, it is seen that an increase in the concentration of the air mixture $(\mu)$ leads to an increase in the fan drive power consumed when using an experimental serial version of the receiving chamber. However, for the serial receiving chamber, the power consumption of the fan drive is 1.66 times higher than the consumption of the experimental receiving chamber. From the graphs in Figure 3, it is seen that an increase in pipeline diameter from $0.15 \mathrm{~m}$ to $0.175 \mathrm{~m}$ decreases the power consumption of the fan drive at $\mu=0.338$ from $10.30 \mathrm{~kW}$ to $5.79 \mathrm{~kW}$, i.e., by 1.78 times when using a serial receiving chamber. With an increase in concentration $\mu=0.228 \div 0.338$ at pipeline diameter $D=0.15 \mathrm{~m}$, the fan drive power increases to $N=9.62 \div 10.30 \mathrm{~kW}$ and, at pipeline diameter $D=0.175 \mathrm{~m}$ it increases to $N=5.42 \div 5.79 \mathrm{~kW}$. With an increase in the pipeline's diameter, the influence of the concentration of the air mixture on the power consumption of the fan drive decreases by $(10.3: 5.79)=1.77$ times.

Figure 4 shows the effect of the change in the pipeline's diameter and the concentration of the air mixture $(\mu)$ on the fan drive power of the experimental receiving chamber. The patterns of graph changes are similar to the patterns in Figure 3; however, the power consumption of the fan drive is 1.65 times less compared to the serial receiving chambers.

\section{Conclusions}

To reduce the power consumption of the fan drive, it is necessary to use a pipeline with a diameter of at least $D=0.175 \mathrm{~m}$. The use of an experimental receiving chamber instead of a serial chamber allows us to reduce the power consumption of the fan drive by 1.65 times. With an increase in the pipeline's diameter from $0.15 \mathrm{~m}$ to $0.175 \mathrm{~m}$, the effect of the air mixture composition on the power consumption of the drive decreases by 1.77 times.

\section{References}

1. Jingshan Tian, Xuyi Zhang, Wangfeng Zhang, Hengyi Dong, Zhan Zhao. Leafadhesivenessaffectsdamagetofiber strength during seed cotton cleaning of machine-harvested cotton. //Industrial Crops and Products, Volume 107, , Pages 211216. November (2017).

2. Kılıçkan A., Güner M.. Pneumatic Conveying Characteristics of Cotton Seeds// Biosystems Engineering, Volume 95, Issue 4, Pages 537-546, December (2006)

3. Cotton: World Statistics. http://www.ICAC. org; http://www.statista.com.

4. Panagiotis A. Ntogkoulis, Dionysis D. Bochtis, Spyros Fountas, Remigio Berruto, Theofanis A. Gemtos. Performance of cotton residue collection machinery / Biosystems Engineering Volume 119 Pages 25-34. March (2014)

5. Yiannis G. Ampatzidis, Stavros G. Vougioukas, Matthew D. Whiting, Qin Zhang. Applying the machine repair model to improve efficiency of harvesting fruit / Biosystems Engineering Volume 120April 2014 Pages 25-33.

6. Mygdakos E., Gemtos T. A.. IT-Information Technology and the Human Interface: Reliability of Cotton Pickers and its Effect on Harvesting Cost / Biosystems Engineering Volume 82, Issue 4 August 2002 Pages 381-391.

7. TIAN Jing-shan1, ZHANG Xu-yi1, ZHANG Wang-feng1, LI Jian-feng1, YANG Yan-long1, DONG Heng-yi2, JIU Xing-li2, YU Yong-chuan3, ZHAO Zhan4, XU Shou-zhen1, ZUO Wen-qing1. Fiber damage of machine-harvested cotton before ginning and after lint cleaning.// Journal of Integrative Agriculture , 17(5): 11201127.(2018) 
8. Rizaev A, Yuldashev A, Kuldoshev D and Ashurov N.Effect parameters of vertically spindle cotton picker machines on the mechanical damage of cotton seeds during machine harvesting./IOP Conf. Series: Materials Science and Engineering 883012166 (2020)

9. Mukhammadiev, D.M. Algorithm development for the pulsator VBFC machine unit simulation./ Journal of Machinery Manufacture and Reliability, 38(1), P. 82-86. (2009)

10. Otazhonov N.S., Matchanov R.F., Rizaev A.A., Malikov Z.M., Yuldashev A.T., et al. Method of transporting raw cotton from the receiving chamber to the bunker of a cotton picker and a device for its implementation / Application No. IAP 20190507. Date of filing (2019).

11. Matchanov R.D., Rizaev A.A., Yuldashev A.T., Kuldoshev D.A., AkhmedovSh.A. Pneumatic transport system of a cotton picker. // International Journal of Innovative Technology and Exploring Engineering (IJTEE). ISSN: 2278-3075, Volume-9 Issue4, - pp.2267-2270. February (2020).

12. A Rizaev, A Yuldashev, D Kuldoshev. Model and calculation development of productivity of cotton harvesters.// IOP Conf. Series: Materials Science and Engineering $919032013 \quad$ IOP Publishing doi:10.1088/1757899X/919/3/032013.(2020).

13. ARizaev, A Yuldashev, D Kuldoshev, $\mathrm{T}$ Abdillaev and $\mathrm{N}$ Ashurov. Advance of spindle drum and frontality of active spindle surface./IOP Conf. Series: Materials Science and Engineering 883 012157doi:10.1088/1757-899X/883/1/012157 (2020).

14. Rizaev A.A., MatchanovR.D., Yuldashev A.T., D Kuldoshev, Djuraeva N.B. Cotton harvesters for one-time cotton-picking. / IOP Conf. Series: Materials Science and Engineering 1030 (2021) 012173 IOP Publishing doi:10.1088/1757899X/1030/1/012173

15. Rizaev A.A., Malikov Z.M.,. Yuldashev A.T, D Kuldoshev, Temirov D.A., Borotov A.N.. Bench-scale study of centrifugal fan parameters./ IOP Conf. Series: Materials Science and Engineering 1030012175 IOP Publishing doi:10.1088/1757899X/1030/1/012175 (2021).

16. Zuev F.G. Pneumatic transportation in grain processing plants. - M.: Kolos, 344 p. (1976).

17. Artykov N.A. Pneumatic transport of easily damaged materials. - Tashkent: Fan, - 152 p. (1984).

18. Idelchik I.E. Reference book on hydraulic resistance. Moscow, Mechanical Engineering, - 559 p. (1975).

19. Topolidi K.G. Pneumatic transport in the textile and light industry. Moscow, Legprofbytizdat, - 91 p. (1987).

20. The program for calculating the pneumatic transport system of the cotton harvesting machine // Matchanov R.D., Rizaev A.A., Yuldashev A.T., Kuldoshev D.A. et al / Patent No. 39615. 